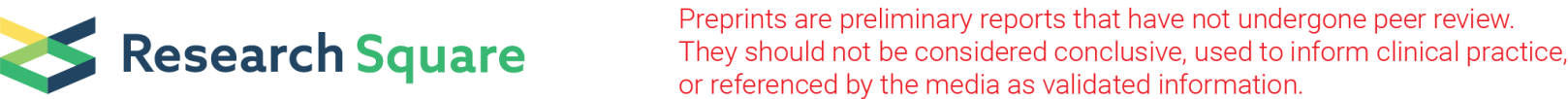

\section{Analysis of Chromosome 3P Genes Associated With Clinical Staging of Small Cell Lung Cancer and Prediction of Targeted Drugs}

\section{Chun-Xu Ma}

Department of Nuclear Medicine, The First Affiliated Hospital of Kunming Medical University Ji-Hua Zhao

Department of Nuclear Medicine, The First Affiliated Hospital of Kunming Medical University

\section{Ying Wu}

Department of Nuclear Medicine, The First Affiliated Hospital of Kunming Medical University

Jun Wang

College of physical education, Yunnan Agricultural University

Hao Wang ( 18810566155@163.com )

Department of Nuclear Medicine, The First Affiliated Hospital of Kunming Medical University

\section{Research Article}

Keywords: Diagnosis, Small-cell lung cancer, Chromosome 3p gene, Quantitative real-time Polymerase Chain Reaction, Drug prediction

Posted Date: November 16th, 2021

DOI: https://doi.org/10.21203/rs.3.rs-1050186/v1

License: (c) (1) This work is licensed under a Creative Commons Attribution 4.0 International License. Read Full License 


\section{Abstract}

\section{Background}

Nowadays, the diagnosis of small-cell lung cancer (SCLC) remains a great challenge. Changes in the chromosome $3 p$ (chr3) gene are usually observed in the pathogenesis of lung cancer, which seems to imply that these chr3 genes may be a diagnostic marker in the early stage of SCLC.

\section{Methods}

Based on Differentially expressed genes (DEGs) screening analysis, Weighted gene co-expression network analysis, and Receiver operating characteristic (ROC) curves, this study explored the diagnostic value of the chr3 gene obtained from the University of California Santa Cruz Genome Browser Database in SCLC. Meanwhile, quantitative real-time Polymerase Chain Reaction (qRT-PCR) was used to reveal the expression patterns of diagnostic biomarkers in Human pulmonary alveolar epithelial cells and the SCLC cell line $\mathrm{NCl}-\mathrm{H} 146$. The potential pathways involved in the diagnostic biomarkers were investigated by a single-gene Gene Set Enrichment Analysis (GSEA). Furthermore, drugs regulating the expression levels of diagnostic biomarkers were predicted by the Comparative Toxicogenomics Database (CTD).

Results

We identified a total of 33 differentially expressed chr3 (DE-chr3) genes and 1156 hub genes associated with clinical features of SCLC, and functional enrichment analysis showed that all of these genes were significantly enriched in the cell cycle terms. Ultimately, the area under curve of the ROC curve demonstrated that the overlapping genes of the DE-chr3 genes and the hub genes, CDC25A, FYCO1, and RFTN1, were relatively accurate in distinguishing normal from SCLC samples, which were considered to be diagnostic biomarkers. CDC25A was overexpressed in SCLC samples, while FYCO1 and RFTN1 were highly expressed in normal samples, as evidenced by qRT-PCR results. Single-gene GSEA showed that diagnostic biomarkers were significantly associated with cell cycle, $A B C$ transporter, immune cell differentiation, immune response, and multiple respiratory disease pathways. Further, a total of 141 drugs were predicted by the CTD to modulate the expression of diagnostic biomarkers, of which 8 drugs were the shared drugs for 3 diagnostic biomarkers.

\section{Conclusions}

This project provided 3 novel and powerful diagnostic biomarkers for SCLC based on the chr3 genes. Meanwhile, a guideline for the development and selection of drugs for clinical treatment based on diagnostic biomarkers was also included.

\section{Background}

Lung cancer accounts for more than one-tenth of all cancer cases worldwide and is one of the most common types of cancer [1]. During 2018, 781,000 new confirmed cases of lung cancer and 626,000 
deaths were reported in China [2]. Specifically, small-cell lung cancer (SCLC), which is characterized by an unusually high proliferation rate, a strong propensity for early metastasis, and a poor prognosis, accounts for approxi-mately $15 \%$ of all lung cancers [3]. The 5 -year survival rate of SCLC patients after di-agnosis was only $2.8 \%$, with a median overall survival (OS) of about 10 months. In ad-dition, Most patients have metastatic disease at diagnosis, with only one-third having an earlier-stage disease, which increased the difficulty of clinical treatment [4]. There-fore, early diagnosis of SCLC is of great significance to improve the OS of SCLC patients.

Currently, no proven effective method exists for the early detection of SCLC. Ac-curate detection of tumor pathological types has been pursued by conventional methods, such as image-guided percutaneous transthoracic needle biopsy and bronchoscopy. However, a biopsy is not suitable for patients with respiratory inadequateness or un-clear location of the tumor. Fortunately, with the continuous development of molecular biology, serum tumor markers have gradually become the diagnostic techniques for malignant tumors including SCLC, such as Neuron Specific Enolase (NSE) and Progastrinreleasing Peptide (ProGRP) [5]. NSE is overexpressed in tumors associated with the origin of neuroendocrine tissue, especially SCLC [6]. ProGRP is significantly elevated in the serum of SCLC patients and is positively correlated with SCLC staging [7]. However, the specificity is not always satisfactory due to the elevated NSE and ProGRP concentration in many patients. In addition, the concentration of NSE in hemolytic samples also increases [8]. Thereby, identifying more reliable diagnostic markers to compensate for the deficiencies of these traditional serum markers is urgently demanded.

Chromosomal copy number variation is a hallmark of cancer [9]. Loss of hetero-zygosity involving several chromosome $3 p$ (chr3) regions accompanied by chr3 dele-tions is detected in almost $100 \%$ of SCLC. In addition, these changes appear early in the pathogenesis of lung cancer. These $3 p$ genetic alterations lead to the conclusion that the short arm of human chr3 contains several tumor suppressor gene(s) [10]. It is clear now that genetic and epigenetic abnormalities of several genes residing in the chr3 region are important for the development of SCLC but it is still obscure how many of them exist and which of the numerous candidate tumor suppressor genes are the key players in SCLC pathogenesis. Therefore, exploring the diagnostic value of the chr3 gene in SCLC will bring an optimistic effect to the clinical diagnosis and prognosis of SCLC.

In this study, gene expression data obtained from Gene Expression Omnibus (GEO) databases were integrated to conduct data mining and analysis of SCLC. Then, a series of co-differentially expressed genes have been screened in SCLC. A series of analyses were carried out based on these genes, including analysis of functional enrichment, single-gene Gene Set Enrichment Analysis (GSEA), drug identification, and human samples validation. We explored the correlation between the genes in chr3 deletion re-gions and the disease progression of SCLC and predicted the therapeutic drugs based on these genes.

\section{Methods}

\section{Data source}


The GSE40275 and GSE60052 datasets involved in this study were downloaded from the GEO database. The GSE40275 dataset (https://www.ncbi.nlm.nih.gov/ geo/query/ acc.cgi?acc=GSE40275) contained 43 cases of normal lung tissue and 21 cases of SCLC lung tissue (Supplementary Table 1). 86 samples were included in the GSE60052 dataset (https://www.ncbi.nlm.nih.gov/geo/query/acc.cgi? acc=GSE60052), including 7 cases of normal lung tissue and 79 tumor samples from SCLC patients (Supplementary Table 2). The GSE40275 dataset was primarily used for screening of Differential expression genes (DEGs), Weighted gene co-expression network analysis (WGCNA), diagnostic biomarker assessment, and single-sample Gene Set Enrichment Analysis (ssGSEA); the GSE60052 dataset was focused on screening of DEGs and diagnostic biomarker assessment.

The 505 chr3 genes (Supplementary Table 3) were obtained from the Human Dec. 2013 (GRCh38/hg38) Assembly in the University of California Santa Cruz (UCSC) Genome Browser Database (https://genome.ucsc.edu).

\section{Analysis of the differential genes}

DEGs were identified in the GSE40275 and GSE60052 datasets respectively using the 'limma' package in the R software. Genes that satisfied $\mid \log 2$ fold change (FD) $\mid>1$ and $P<0.05$ between normal and SCLC samples were considered as DEGs. The over-lapping genes of DEGs (co-DEGs) in the above two datasets were obtained by a Venn analysis, which was performed on the jvenn online website (http://jvenn.toulouse. inra.fr/app/example.html). Working as usual, we also identified DE-chr3 genes (overlapping genes of co-DEGs and chr3 genes). Furthermore, the visualization and embellishment of the Venn diagrams were implemented in the jvenn online website.

\section{Metascape}

Metascape (http://metascape.org/) is a powerful tool for functional annotation analysis of genes [11]. In this study, we used this tool to perform comprehensive functional enrichment analysis of the DE-chr3 gene, including Gene Ontology (GO), Kyoto Encyclopedia of Genes and Genomes (KEGG), and Reactome analysis. GO analysis was based on three components: cellular component (CC), molecular function (MF), and biological process (BP) [12]. KEGG [13] and Reactome [14] analyses were used to explore the pathways in which genes might be involved. Terms at $P<0.01$ were considered significantly enriched.

\section{Ingenuity pathway analysis (IPA)}

IPA is a cloud-based, graphical bioinformatics software that mines genomic data for hidden biological significance from a biological pathway perspective. The Ingenuity Knowledge Base (IPKB), the core component of IPA, is a specialized biological interaction and functional annotation database that contains millions of information on the most direct interactions between proteins, genes, compounds, cells, tissues, drugs, and diseases. In this study, we uploaded the DE-chr3 genes to IPKB, focusing on the results of its Disease \& Function analysis. 
We extracted genes with mean expression values (FPKM) >1 from all SCLC samples (GSE40275 dataset) to perform WGCNA using clinical and expression data from the GSE40275 dataset. The clinical traitrelated modules were constructed and hub genes were identified in the WGCNA package. Briefly, we performed a hierarchical cluster analysis on the GSE40275 dataset samples to exclude outliers (GSM990246 sample was the outlier and excluded; Supplementary Fig. 1A). Here, the soft threshold power was set to 11 (scale-free $R^{2}=0.85$ ). Modules were segmented by the dynamic tree-cutting algorithm, and MEDissThres was set to 0.5 to merge similar modules (Supplementary Fig. 1B to D). Finally, correlations between module genes and clinical features were calculated. The genes in the modules that were most correlated with clinical features were considered to be hub genes. It should be noted that because the M-stage information for patients recorded in the GSE40275 dataset was all MX, M-stage was not considered. Therefore, clinical characteristics included pathological T and N stages (pT, $\mathrm{pN}$ ), tumor stage (stage), sex, and age. Detailed functional annotation of hub genes was worked out by GO and KEGG enrichment analysis in the cluster Profiler package.

\section{Receiver operating characteristic (ROC) curves and area under curve (AUC) estimation based on diagnostic biomarkers}

Similarly, as previously described, the overlapping genes of the hub and DE-chr3 genes were obtained by Venn analysis to use them as candidate biomarkers. Subsequently, ROC curves and corresponding AUCs were established in the GSE40275 and GSE60052 datasets to evaluate the ability of candidate biomarkers to correctly diagnose disease. Only candidate biomarkers with an AUC $>0.85$ in both datasets were identified as diagnostic biomarkers for SCLC. Moreover, expression profiles were extracted from the GSE40275 and GSE60052 databases to demonstrate the expression patterns of the selected biomarkers.

\section{Cell culture}

Human pulmonary alveolar epithelial cells (HPAEpiC) and SCLC cell line NCl-H146 were purchased from the American Type Culture Collection (ATCC). The HPAEpiC was maintained in Dulbecco's modified eagle medium (DMEM; Hyclone, Logan, UT, USA) supplemented with 10\% fetal bovine serum (FBS; Gibco, Invitrogen, Paisley, UK) and 1\% penicillin-streptomycin (Lonza, Verviers, Belgium). NCl-H146 was grown in the Roswell Park Memorial Institute (RPMI)-1640 medium (Sigma-Aldrich, St. Louis, MO, USA) supplemented with $10 \% \mathrm{FBS}, 1 \%$ penicillin-streptomycin. The cells were grown at $37^{\circ} \mathrm{C}$ in a humidified atmosphere of $95 \%$ air and $5 \% \mathrm{CO}_{2}$. The experiments were carried out on cells whose passage was between 10 and 25 .

\section{RNA extraction and quantitative real-time polymerase chain reaction (qRT-PCR)}

Total RNA was extracted from all HPAEpiC and NCl-H146 using TRIzol reagent (Invitrogen, Carlsbad, CA, USA). The concentration and quality of RNA were deter-mined by spectrophotometry (Jinghua Technology, Shanghai, China) at $260 \mathrm{~nm}$ and $280 \mathrm{~nm}$. Total RNA was reverse transcribed using the 
SureScript-First-strand-cDNA-synthesis-kit (Genecopoeia, Guangzhou, China) according to the manufacturer's instructions. qRT-PCR was performed using CFX96 real-time PCR detection (CFX96; BioRad, USA) systems using the BlazeTaq ${ }^{\text {TM }}$ SYBR ${ }^{\circledR}$ Green qPCR Mix 2.0 kit (Genecopoeia, Guangzhou, China) according to manufacturer's instructions. Relative expression values were calculated using the 2$\triangle \mathrm{CT}$ method [15]. The primers were synthesized by Tsingke Biotech Co., Ltd. (Shanghai, China), and sequences are listed in Table 1.

\section{Single-gene GSEA}

In this section, single-gene GSEA was applied to analyze the pathways enriched by each diagnostic biomarker. Briefly, samples were divided into high- and low-expression groups using the median expression of each diagnostic biomarker in the GSE40275 dataset. Subsequently, $\log _{2}$ FC values were calculated for all genes between the high- and low-expression groups for each diagnostic biomarker (please noted that DEGs between high- and low-expression groups were not defined here, only the difference between the two groups was analyzed) and sorted from highest to lowest according to this value, and the sorted genes were used as the set of genes to be tested; simultaneously, we used the KEGG pathway as a pre-defined set of genes. Finally, single-gene GSEA was performed separately for each diagnostic biomarker in the cluster Profiler package to detect the enrichment of the pre-defined gene set in the set of genes to be tested. Adjusted (adj.) $P<0.05$ for the pathway was significant.

\section{Drug identification by the CTD}

The CTD (http://ctdbase.org/) is a scientific database for describing the relationship between chemicals, genes, and human diseases. Given a gene, the CTD can provide the corresponding target compounds in descending order of their interactions. In this study, CTDs with default parameters were used to predict the candidate drugs for the selected biomarkers.

\section{Statistical analysis}

The bioinformatic analysis was carried out in the R software. The complete drug-diagnostic biomarker network was visualized by Cytoscape software (v 3.6.1; https://www.nigms.nih.gov/). For shared drugs, intersection analysis was performed on the jvenn online website to obtain the results. Subsequent shared drug-diagnostic bi-omarker networks were drawn and embellished in the diagrams.net online network (https://app.diagrams.net/). The histogram was constructed using GraphPad Prism 8 (GraphPad Software, La Jolla, CA) software to indicate the relationship between tissue type (normal and SCLC) and mRNA levels of diagnostic biomarkers. If not otherwise stated, $P<0.05$ was considered as the threshold for statistical significance.

\section{Results}

\section{Identification of the DE-chr3 gene related to SCLC}


In the GSE40275 dataset, we identified a total of 2189 DEGs from between 21 SCLC and normal samples, of which 1115 were up-regulated and 1074 were down-regulated (Fig. 1A; Supplementary Table 4). Meanwhile, 6290 DEGs were extracted from the GSE60052 dataset (79 SCLC vs. 7 normal), including 2720 up-regulated genes and 3572 down-regulated genes (Fig. 1B; Supplementary Table 5). The Venn analysis indicated that a total of 1162 co-DEGs (407 up-regulated genes, 755 down-regulated genes) were differentially expressed between SCLC samples and normal samples in the two datasets mentioned above (Fig. 1C; Supplementary Table 6). Subsequently, 505 genes located in chr3:1-90000000 were obtained by Human Dec. 2013 (GRCh38/hg38) Assembly in the University of California Santa Cruz (UCSC) Genome Browser Database, designating them as chr3 genes (Supplementary Table 3). Fig. 1D revealed that only 33 of the 505 chr3 genes belonged to co-DEGs, of which 5 were up-regulated and 28 were down-regulated (Supplementary Fig. 2A and B; Supplementary Table 7).

Furthermore, based on Metascape-GO analysis, we found that the DE-chr3 genes were tightly associated with oxygen metabolic process ('reactive oxygen species metabolic process' and 'cellular response to oxidative stress'), cell motility ('negative regulation of epithelial cell migration', 'ameboid-type cell migration', 'endothelial cell migration', etc.), and 'the regulation of inflammatory responses'. Surprisingly, the 'regulation of supramolecular fiber organization' and 'supramolecular fiber organization' were found to be significantly enriched (Supplementary Fig. 2C to F; Supplementary Table 8). Several supercoiled supramolecular polymeric fibres of self-sorted donor-acceptor molecules (supramolecular fibres) have been reported as markers of lung cancer [16]. Besides, IPA's disease \& function analysis pointed out that these DE-chr3 genes were involved not only in cellular, tissue, and organ development but also in cancer, tumor morphology, and respiratory diseases (Supplementary Table 9). This evidence suggested that the DE-chr3 genes were probably playing an essential role in the development and progression of SCLC.

\section{Searching for hub genes associated with clinical features of SCLC by WGCNA}

WGCNA was performed in 20 SCLC samples from the GSE40275 dataset. With a soft threshold set to 11 $\left(R^{2}=0.85\right)$, the gene network infinitely approximated the scale-free distribution (Fig. 2A). Twelve coexpressed modules were then identified (Fig. 2B). Correlations between modules and clinical characteristics were calculated, including age, sex, $\mathrm{pT}, \mathrm{pN}$, and stage. The dark grey module was strongly affiliated with pT $(r=0.59, P=0.006), \mathrm{pN}(r=0.59, P=0.006)$, sex $(r=0.6, P=0.005)$, and stage $(r=0.75$, $P=1 \mathrm{e}-04)$. Therefore, the dark grey module was selected as the hub module, of which 1156 genes (Supplementary Table 10) would be investigated in the subsequent analysis.

The potential functions of these hub genes were also explored. GO-BP analysis revealed that these genes were significantly enriched in various cell cycle-related terms, including but not limited to 'regulation of cell cycle G2/M phase transition', 'mitotic sister chromatid segregation', 'organelle fission', 'ribonucleotide metabolic process', and 'spindle checkpoint' (Fig. 2C; Supplementary Table 11). Similarly, the KEGG pathway prompted that the cell cycle pathway was also significantly enriched (Fig. 2D; Supplementary 
Table 12). This evidence recommended that the variation in cell cycle processes might be relevant to the course of SCLC.

\section{Identification and assessment of diagnostic biomarkers for SCLC}

To further identify biomarkers for SCLC, we obtained three overlapping genes for the DE-chr3 genes and the hub genes, CDC25A, FYCO1, and RFTN1, respectively (Fig. 3A). The ability of the three overlapping genes to discriminate between normal and SCLC samples was subsequently assessed by ROC curves (Fig. 3B and C). Abbreviately, CD25A had an AUC of 0.988 in the GSE40275 dataset and 0.880 in the GSE60052 dataset; FYCO1 had AUCs of 1 and 0.944 in the GSE40275 and GSE60052 datasets, respectively; and RFTN1 had AUCs of 0.993 and 0.868 in the above two datasets, respectively. This evidence suggested that the three overlapping genes possessed a robust capacity to differentiate from the normal and SCLC groups, which were considered as diagnostic biomarkers for SCLC in the follow-up analysis. Thereby, CDC25A was the gene that was up-regulated in SCLC, whereas FYCO1 and RFTN1 were overexpressed in normal samples, and the expression patterns of these genes were consistent in the GSE40275 (Fig. 3D) and GSE60052 (Fig. 3E) datasets. Consistently, qRT-PCR results (Fig. 3F) pointed out that $C D C 25 A$ exhibited a higher expression level in NCl-H146 compared to HPAEpiC $(P<0.0001)$, whereas FYCO1 and RFTN1 were significantly decreased in NCl-H146 ( $P=0.0421$ and $P=0.0005$, respectively).

Additionally, we examined the correlation between the expression of diagnostic biomarkers and the clinical characteristics of SCLC in the GSE40275 dataset. The results presented that all 3 genes were significantly associated with pT, stage, and sex. Briefly, CDC25A was markedly overexpressed in pT4, stage IIIB, and male patients (Supplementary Fig. 3A); RFTN1 was grossly upregulated in pT4, stage III, and male patients (Supplementary Fig. 3B); FYCO1 was overexpressed in pT2, stage I, and female patients and pNO patients had a higher expression of FYCO1 (Supplementary Fig. 3C).

\section{Pathway enrichment analysis of each diagnostic biomarker}

To reveal the potential pathways involved in the diagnostic biomarkers, we performed a single-gene GSEA for each diagnostic biomarker in the GSE40275 dataset. The results revealed that CDC25A was enriched to a total of 22 KEGG pathways (Fig. 4A; Supplementary Table 13); RFTN1 was significantly associated with 52 KEGG pathways (Fig. 4B; Supplementary Table 14); and FYCO1 was mainly involved in 8 KEGG pathways (Fig. 4C; Supplementary Table 15).

Specifically, all 3 diagnostic biomarkers were implicated in the KEGG pathway of 'Cell cycle', 'DNA replication', and 'Homologous recombination'. This suggested that diagnostic biomarkers might also be involved in the proliferation process of tumor cells. Interestingly, the 'ABC transporter' pathway was significantly enriched in $C D C 25 A$ and FYCO1. The ABC transporter superfamily is known to be a family of membrane transporter proteins whose increased intracellular drug pumping is thought to be one of the mechanisms of tumor multidrug resistance, while many members of the ABC superfamily have also been shown to be involved in tumor multidrug resistance [17-19]. We also found that the 'Drug metabolism- 
other enzymes' pathway was notably associated with RFTN1. Such evidence indicated that diagnostic biomarkers might bring new hope for the development of novel drugs for SCLC. Notably, we also found that immune cells ('Th17 cell differentiation', 'Th1 and Th2 cell differentiation', 'Natural killer cell mediated cytotoxicity', 'Neutrophil extracellular trap formation') and immune response ('Antigen processing and presentation', 'Cell adhesion molecules', 'Chemokine signaling pathway', 'Fc epsilon RI signaling pathway', 'Rap1 signaling pathway')-related pathways would appear to be inextricably linked to RFTN1. The reason why SCLC is reportedly not sensitive to immunotherapy is that it lacks a surface protein that triggers the immune response, and this innate deficiency may also be related to tumor immune escape in SCLC [20]. Therefore, we hypothesized that RFTN1 might provide a theoretical basis and direction for finding effective immunotherapeutic targets and mechanisms for SCLC. Besides, several respiratory diseases were also highlighted, such as 'Asthma' (CDC25A and RFTN1), 'Tuberculosis' (RFTN1), 'Influenza A' (RFTN1), 'Coronavirus disease-COVID-19' (RFTN1), and 'Pertussis' (RFTN1).

\section{Pharmaceutical prediction for regulating the expression of diagnostic biomarkers}

Inspired by the above results, CTD [21] was employed to predict potential pharmaceutical agents that could modulate the expression of diagnostic biomarkers, as described in the Materials and Methods section. A total of 141 potential drugs were identified by CTD (Supplementary Table 16). A complete drugdiagnostic biomarker network was constructed using Cytoscape, containing 144 nodes with 223 edges (Fig. 5A). The smaller circles represented the predicted drugs and the larger circles were diagnostic biomarkers. The red, green, and grey lines indicated up-regulation, down-regulation, and both upregulation and down-regulation, respectively. Afterward, we presented the regulatory relationships of 27 shared drugs (drugs shared by two diagnostic biomarkers and drugs shared by all three diagnostic biomarkers) with diagnostic biomarkers in Fig. 5B. Where circles represented diagnostic biomarkers; rounded rectangles indicated predicted drugs (brown for drugs shared by CDC25A and FYCO1, orange for drugs shared by FYCO1 and RFTN1, purple for drugs shared by CDC25A and RFTN1, and yellow for drugs shared by all three diagnostic biomarkers); blue straight lines represented down-regulation; red straight lines represented up-regulation; green straight lines represented both up- and down-regulation. Acetaminophen, Cisplatin, Coumestrol, Estradiol, Sunitinib, Valproic Acid, 2,3-bis (3'-hydroxybenzyl) butyrolactone, JinFuKang were the shared drugs for three diagnostic biomarkers whose potential guided the development of new drugs for SCLC (Table 2).

\section{Table 1 Primer sequences for qRT-PCR.}

The table should appear at the end of "RNA extraction and quantitative real-time polymerase chain reaction (qRT-PCR)" in "Methods". 


\begin{tabular}{|lll|}
\hline Genes & Forward primer $\left(\mathbf{5}^{\prime} \mathbf{3}^{\prime} \mathbf{)}\right.$ & Reverse primer $\left(\mathbf{5}^{\prime} \mathbf{3}^{\prime} \mathbf{)}\right.$ \\
\hline CDC25A & TATGAGCAACCACTGGAGGT & GTGACTGGGGTGAAAAAGA \\
\hline FYCO1 & GTGGGGCAGGATTCGGAAAT & TGGGGATCAGGCTGTAGGTG \\
\hline RFTN1 & TTCCTCCTTAGACCACCCGA & AGTTCTCCACCATCTCCCTC \\
\hline Gapdh & CGCTGAGTACGTCGTGGAGTC & GCTGATGATCTTGAGGCTGTTGTC \\
\hline
\end{tabular}

Table 2 The 8 shared predicted drugs for three diagnostic biomarkers and their interactions.

The table should appear at the end of the "results". 


\begin{tabular}{|c|c|c|c|c|}
\hline \multirow[t]{2}{*}{ Group } & \multirow[t]{2}{*}{ Predicted Drugs } & \multicolumn{3}{|c|}{ Interactions } \\
\hline & & CDC25A & FYCO1 & \\
\hline \multirow[t]{5}{*}{ 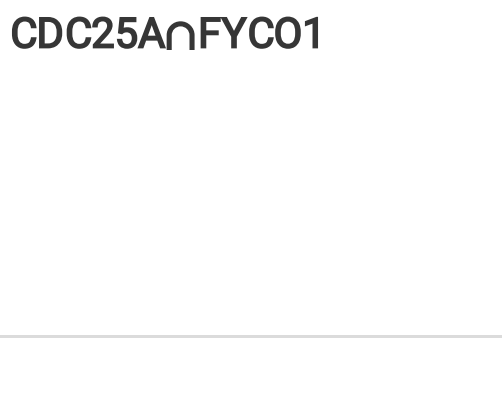 } & Arsenic Trioxide & UP & UP & \\
\hline & bisphenol A & DN & UP & \\
\hline & Copper Sulfate & DN & UP & \\
\hline & Rotenone & DN & UP & \\
\hline & & CDC25A & RFTN1 & \\
\hline \multirow[t]{13}{*}{ CDC25AกRFTN1 } & Aflatoxin B1 & UP & DN & \\
\hline & (+)-JQ1 compound & UP/DN & DN & \\
\hline & Tetrachlorodibenzodioxin & UP/DN & UP/DN & \\
\hline & Tobacco Smoke Pollution & UP/DN & DN & \\
\hline & Arsenic & UP & UP & \\
\hline & sodium arsenate & UP & UP & \\
\hline & Tamoxifen & UP & DN & \\
\hline & Cyclosporine & DN & DN & \\
\hline & Hydrogen Peroxide & DN & UP & \\
\hline & tris(1,3-dichloro-2-propyl)phosphate & DN & DN & \\
\hline & Resveratrol & UP & UP & \\
\hline & Copper & DN & UP & \\
\hline & & FYCO1 & RFTN1 & \\
\hline \multirow[t]{4}{*}{ FYC01กRFTN1 } & abrine & DN & UP & \\
\hline & methylmercuric chloride & DN & UP & \\
\hline & Urethane & DN & DN & \\
\hline & & CDC25A & FYCO1 & RFTN1 \\
\hline \multirow[t]{5}{*}{ 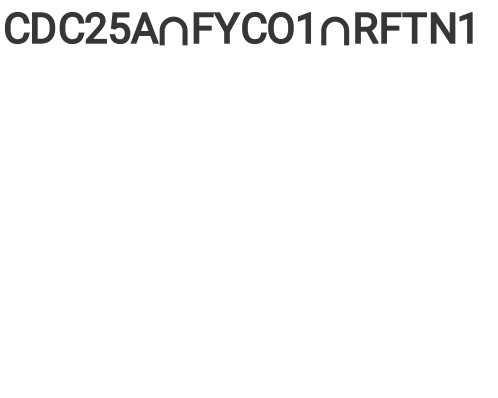 } & Acetaminophen & UP & DN & DN \\
\hline & Cisplatin & UP/DN & UP & DN \\
\hline & Coumestrol & UP & DN & DN \\
\hline & Estradiol & UP & UP & UP \\
\hline & Sunitinib & DN & UP & UP \\
\hline
\end{tabular}




\begin{tabular}{|llll|} 
Valproic Acid & DN & UP & UP/DN \\
\hline 2,3-bis(3'-hydroxybenzyl)butyrolactone & UP & DN & DN \\
\hline jinfukang & DN & UP & DN \\
\hline
\end{tabular}

\section{Discussion}

SCLC is the most aggressive form of lung cancer. Compared with NSCLC, SCLC is characterized by a rapid doubling time and early, widespread metastases. Lack of early detection modalities is one of the significant barriers to progress in diagnosis and treatment of SCLC. Therefore, novel biomarkers with high efficiency, high sensitivity, and high specificity are urgently needed for the diagnosis and prognosis of SCLC. Pre-vious studies have shown that chr3 alterations may be associated with the pathogenesis of SCLC. So chr3 genes present the promising potential of becoming a diagnostic bi-omarker of SCLC.

The development of high-throughput sequencing facilitates the search for poten-tially involved genes and tumor mechanisms. Bioinformatics, on the other hand, uses genetic information for molecular diagnoses and drug therapies [22]. Integrated bioin-formatics analysis for the identification of therapeutic targets of certain cancers based on transcriptomics, proteomics, and high-throughput sequencing could help us obtain novel information and understand potential underlying molecular mechanisms. In the current study, we identified $33 \mathrm{DE}$-chr3 genes which may play an essential role in the development and progression of SCLC and confirmed that the variation in cell cycle processes might be relevant to the course of SCLC by GO-BP analysis. Besides, we ob-tained 3 overlapping genes for the DE-chr3 genes and the hub genes, CDC25A, FYCO1, and RFTN1, respectively by bioinformatics functional assessment.

CDC25A is one of the cell cycle regulation-related genes. CDC25A is mainly local-ized in the nucleus, and controls $\mathrm{G} 1 / \mathrm{S}$ progression by dephosphorylation-dependent inactivation of Cyclin E/CDK2 and Cyclin D/CDK4-6; G2 phase progression by dephosphorylation of CDK1 and activation of cyclin B1/CDK1 complex is a limiting step [23]. A study suggested that CDC25A controls the cell proliferation and tumorigenesis by a change in expression of proteins involved in cyclin D1 regulation and G1/S transition [24]. Maintenance of proper $C D C 25 A$ levels is important for genomic stability and tumor suppression [25]. The activity and abundance of $C D C 25 A$ are intricately regulated and $C D C 25 A$ is frequently overexpressed in several cancer Types [26-29]. Butz $\mathrm{H}$ et al [30] revealed that the overexpression of $C D K 1$ and $C D C 25 A$ may have an important role in promoting pituitary tumors in the $\mathrm{G} 2 / \mathrm{M}$ transition phase. Our study showed that the expression of CDC25A in SCLC was significantly increased, and we speculated that the high expression of CDC25A in SCLC may also promote the occurrence and development of SCLC during the $\mathrm{G} 2 / \mathrm{M}$ transition phase. But the specific mechanism needs to be further explored. Rao PC et al [31] discovered that aberrant expression of the cell cycle regulation-related genes Cyclin D1, Cyclin E, Cyclin A, $C D C 25 A$, and $C D K 4$ could facilitate the transcription and expression of genes that are associated with cell cycle progression. One study suggested that the cell cycle pathway would be the pathway most closely associated with the pathogenesis of SCLC [22]. However, the functions of the cell cycle and its regulatory proteins in SCLCs have not been fully clarified. 
FYCO1 is a late Rab7 effector of autophagy that is required for the maturation of autophagosomes [32]. FYCO1 has been reported to link autophagosome to microtubule (MT) plus-end movement motor kinesin, which promotes maturation of autophago-some and formation of autophagolysosome [32]. Dionne LK et al discovered that FYCO1 regulates the accumulation of post-mitotic midbodies by mediating LC3dependent midbody degradation [33]. It was reported as one of the key genes involved in adenoma to carcinoma transition in colorectal cancer [34]. Another study showed that the expression levels of FYCO1 in paired bladder tissue and urine samples were significantly lower in bladder cancer than in the control group [35]. Lipid raft linker 1 (RFTN1) has been studied in many diseases. Wang $\mathrm{J}$ et al concluded that RFTN1 may be involved with the pathogenesis of glaucoma [36]. Another study showed that RFTN1 may participate in smoking behavior through modulating immune responses or interactions with the glucocorticoid receptor alpha and the androgen receptor [37]. Zhao $Y$ et al explored molecular subtypes and core genes for lung adenocarcinoma and screened out two core genes including CNTN4 and RFTN1. They concluded that low expression of CNTN4 and RFTN1 predicted poorer clinical outcomes for lung adenocarcinoma patients [38]. The above studies proved that FYCO1 and RFTN1 are involved in the occurrence and development of a variety of tumors and many other diseases. However, the specific roles of CNTN4 and RFTN1 in the pathogenesis of SCLC have not been elucidated.

In our study, we found that FYCO1 and RFTN1 were overexpressed in normal samples. Besides, all 3 genes were significantly associated with pT, stage, and sex of SCLC. These results indicated that the 3 overlapping genes may play an important role in the development of SCLC. The results of single-gene GSEA for each diagnostic biomarker revealed the potential pathways involved in the diagnostic biomarkers, such as 'proliferation process of tumor cells', 'immune cells', and 'immune response'. The specific roles of different diagnostic biomarkers in different pathways need to be further studied and explored. It provides hope for SCLC patients to find novel targeted drugs to improve therapeutic effect and prolong their survival time.

Here, pharmaceutical prediction for regulating the expression of diagnostic bi-omarkers was carried out based on CTD. The results revealed that Acetaminophen, Cisplatin, Coumestrol, Estradiol, Sunitinib, Valproic Acid, 2,3-bis (3'-hydroxybenzyl) butyrolactone, JinFuKang were the shared drugs for 3 diagnostic biomarkers whose potential guided the development of new drugs for SCLC. Some of these drugs have been studied in lung cancer in previous studies. A Large Cohort Study showed that total NSAID use was associated with a small reduced risk of lung cancer, which suggested that NSAIDS may be useful for chemoprevention [39]. Cisplatin, a well-known chemotherapeutic drug, has been used for the treatment of numerous human cancers including bladder, head and neck, lung, ovarian, and testicular cancers [40]. Cisplatin exerts anticancer activity via multiple mechanisms but its most acceptable mechanism involves the generation of DNA lesions by interacting with purine bases on DNA fol-lowed by activation of several signal transduction pathways which finally lead to apoptosis [41]. Coumestrol is a natural compound exhibiting broad anti-cancer effects against skin melanoma, lung cancer, and colon cancer cell growth. The anti-cancer effect of coumestrol is due to the direct targeting of haspin kinase [42]. Que $Z$ et al [43] confirmed that Jingfukang induces anti-cancer activity through oxidative stress-mediated DNA damage in circulating human lung cancer cells. Platta CS et al [44] discovered that the HDAC inhibitor valproic acid 
activates Notch1 signaling in SCLC cells and induces changes in cell morphology and suppresses neuroendocrine tumor markers. Additionally, valproic acid profoundly inhibits SCLC cell growth. Hubaux R et al [45] demonstrate valproic acid improves the efficacy of a second-line regimen (vindesine, doxorubicin, and cyclophosphamide) in SCLC cells and in mouse models. The advances of the past decades in defining the genetics and biological pathways driving SCLC have identified multiple novel therapeutic strategies. Current and future efforts are underway to explore combinations of immunotherapies, small-molecule, and chemotherapy combinations with immunotherapy, as well as biomarkers for the selection of immunotherapies.

There were limitations to the present study. First, the conclusions were drawn based on data from public databases rather than actual experiments, meaning that the quality of the data cannot be guaranteed and that the results may be inaccurate. Secondly, the propensity of SCLC to metastasize extensively early in the course of the disease (most commonly to the brain, liver, or bone) leads to a $95 \%$ mortality rate. But $M$-stage was not considered in our study because the M-stage information for patients recorded in the GSE40275 dataset was all MX. It may affect the comprehensiveness of results in our study. Finally, due to the lack of public data on the prognosis of patients with SCLC, the impact of the new drugs on survival was not further investigated. All of these factors could affect the conclusions of the present study.

\section{Conclusions}

The present study has revealed 3 novel and powerful diagnostic biomarkers for SCLC based on the chr3 genes: CDC25A, FYCO1, and RFTN1. All 3 genes were significantly associated with $\mathrm{pT}$, stage, and sex. Meanwhile, a guideline for the development and selection of drugs for clinical treatment based on diagnostic biomarkers was also included. The 3 diagnostic biomarkers may potentially guide the development of new drugs for SCLC. We hope that our findings may offer new perspectives for patients with SCLC in future research and clinical applications. However, more well-designed experiments with larger sample sizes need to be performed to confirm these conclusions.

\section{Abbreviations}

SCLC: small-cell lung cancer; OS: overall survival; NSE: Neuron Specific Enolase; ProGRP: Progastrinreleasing Peptide; chr3: chromosome 3p; GEO: Gene Expression Omnibus; GSEA: Gene Set Enrichment Analysis; DEGs: Differential expression genes; WGCNA: Weighted gene co-expression network analysis; sSGSEA: single-sample Gene Set Enrichment Analysis; UCSC: University of California Santa Cruz; GO: Gene Ontology; KEGG: Kyoto Encyclopedia of Genes and Genomes; IPKB: Ingenuity Knowledge Base; ROC: receiver operating characteristic; AUC: area under curve; HPAEpiC: Human pulmonary alveolar epithelial cells; ATCC: American Type Culture Collection; qRT-PCR: quantitative real-time polymerase chain reaction

\section{Declarations}


Acknowledgements

We sincerely express our gratitude to Huai Ning for his providing language help.

\section{Funding}

This research was funded by National Nature Science Foundation (grant number 81960320), the Priority Union Foundation of Yunnan Provincial Science and Technology Department and Kunming Medical University (grant number 202001AY070001-199) and Yunnan health training project of high level talents (grant number D-2019025).

\section{Availability of data and materials}

The datasets supporting the conclusions of this article are included within the article.

\section{Ethics approval and consent to participate}

Not applicable.

\section{Competing interests}

The authors declare that they have no competing interests.

\section{Consent for publication}

Not applicable.

\section{Authors' contributions}

1. Conceptualization: Hao Wang and Jun Wang. (2) Methodology: Chun-Xu Ma and

Ji-Hua Zhao. (3) Software: Jun Wang. (4) Validation: Chun-Xu Ma; Ji-Hua Zhao and Ying Wu. (5) Formal analysis: Ying Wu. (6) Investigation: Chun-Xu Ma and Ying Wu. (7) Resources: Hao Wang. (8) Data curation: Chun-Xu Ma and Ji-Hua Zhao. (9) Writing-original draft preparation: Chun-Xu Ma. (10) Writingreview and editing: Ji-Hua Zhao; Hao Wang and Jun Wang. (11) Visualization: Jun Wang. (12) Supervision: Hao Wang and Jun Wang. (13) Project administration: Hao Wang and Jun Wang. All authors have read and agreed to the published version of the manuscript.

\section{Authors' information}

Chun-Xu Ma: pony201@qq.com; Ji-Hua Zhao: zhaojihua415@163.com;

Ying Wu: 2272840674@qq.com; Jun Wang: 158291721@qq.com;

Hao Wang: 18810566155@163.com. 


\section{Author details}

${ }^{1}$ Department of Nuclear Medicine, The First Affiliated Hospital of Kunming Medical University, Kunming, China. ${ }^{2}$ College of physical education, Yunnan Agricultural University, Kunming, China.

\section{References}

1. Semenova EA, Nagel R, Berns A. Origins, genetic landscape, and emerging therapies of small cell lung cancer. Genes Dev. 2015;29(14):1447-62.

2. Bray F, Ferlay J, Soerjomataram I, Siegel RL, Torre LA, Jemal A. Global cancer statistics 2018 :

GLOBOCAN estimates of incidence and mortality worldwide for 36 cancers in 185 countries. CA cancer J Clin. 2018;68(6):394-424.

3. Rudin CM, Brambilla E, Faivre-Finn C, Sage J. Small-cell lung cancer. Nat Rev Dis Primers. 2021;7(1): 3.

4. Nicholson AG, Chansky K, Crowley J, Beyruti R, Kubota K, Turrisi A, et al. The International Association for the Study of Lung Cancer Lung Cancer Staging Project: Proposals for the Revision of the Clinical and Pathologic Staging of Small Cell Lung Cancer in the Forthcoming Eighth Edition of the TNM Classification for Lung Cancer. J Thorac Oncol. 2016;11(3):300-11.

5. Zamay TN, Zamay GS, Kolovskaya OS, Zukov RA, Petrova MM, Gargaun A, et al. Current and Prospective Protein Biomarkers of Lung Cancer. Cancers (Basel). 2017;9(11): 155.

6.Bonner JA, Sloan JA, Rowland KM, Klee GG, Kugler JW, Mailliard JA, et al. Significance of neuronspecific enolase levels before and during therapy for small cell lung cancer. Clin Cancer Res. 2000;6(2):597-601.

7. Lamy PJ, Grenier J, Kramar A, Pujol JL. Pro-gastrin-releasing peptide, neuron specific enolase and chromogranin A as serum markers of small cell lung cancer. Lung Cancer. 2000;29(3):197-203.

8. Liu LJ, Teng JL, Zhang LJ, Cong PS, Yao Y, Sun GR, et al. The Combination of the Tumor Markers Suggests the Histological Diagnosis of Lung Cancer. Biomed Res Int. 2017; 2017: 2013989.

9. Li YL, Roberts ND, Wala JA, Shapira O, Schumacher SE, Kumar K, et al. Patterns of somatic structural variation in human cancer genomes. Nature. 2020;578(7793): 112-121.

10.Zabarovsky ER, Lerman MI, Minna JD. Tumor suppressor genes on chromosome $3 p$ involved in the pathogenesis of lung and other cancers. Oncogene. 2002;21(45): 6915-35.

11. Zhou YY, Zhou B, Pache L, Chang M, Khodabakhshi AH, Tanaseichuk O, et al. Metascape provides a biologist-oriented resource for the analysis of systems-level datasets. Nat Commun. 2019;10(1):1523. 
12. The Gene Ontology Consortium. Expansion of the Gene Ontology knowledgebase and resources. Nucleic Acids Res. 2017; 45(D1): D331-D338.

13. Kanehisa M, Goto S. KEGG: kyoto encyclopedia of genes and genomes. Nucleic Acids Res. 2000;28(1):27-30.

14. Fabregat A, Jupe S, Matthews L, Sidiropoulos K, Gillespie M, Garapati P, et al. The Reactome Pathway Knowledgebase. Nucleic Acids Res. 2018;46(D1): D649-D655.

15. Schmittgen TD, Livak KJ. Analyzing real-time PCR data by the comparative $C(T)$ method. Nat Protoc. 2008;3(6):1101-1108.

16. Sandeep A, Praveen VK, Kartha KK, Karunakaran V, Ajayaghosh A. Supercoiled fibres of self-sorted donor-acceptor stacks: a turn-off/turn-on platform for sensing volatile aromatic compounds. Chem Sci. 2016;7(7):4460-4467.

17. Li W, Zhang H, Assaraf YG, Zhao K, Xu XJ, Xie JB, et al. Overcoming ABC transporter-mediated multidrug resistance: Molecular mechanisms and novel therapeutic drug strategies. Drug Resist Updat. 2016;27:14-29.

18. Chauncey TR. Drug resistance mechanisms in acute leukemia. Curr Opin Oncol. 2001;13(1):21-26.

19. Pérez-Tomás R. Multidrug resistance: retrospect and prospects in anti-cancer drug treatment. Curr Med Chem. 2006;13(16):1859-1876.

20. Zhu MR, Huang Y, Bender ME, Girard L, Kollipara R, Eglenen-Polat B, et al. Evasion of Innate Immunity Contributes to Small Cell Lung Cancer Progression and Metastasis. Cancer Res. 2021;81(7):1813-1826.

21. Davis AP, Grondin CJ, Johnson RJ, Sciaky D, Wiegers J, Wiegers TC, et al. Comparative Toxicogenomics Database (CTD): update 2021. Nucleic Acids Res. 2021;49(D1): D1138-D1143.

22. Liao Y, Yin GF, Wang X, Zhong P, Fan XM, Huang CL. Identification of candidate genes associated with the pathogenesis of small cell lung cancer via integrated bioinformatics analysis. Oncol Lett. 2019;18(4): 3723-3733.

23.Shen $\mathrm{T}$, Huang S. The role of Cdc25A in the regulation of cell proliferation and apoptosis. Anticancer Agents Med Chem. 2012;12(6):631-9.

24. Sadeghi H, Golalipour M, Yamchi A, Farazmandfar T, Shahbazi M. CDC25A pathway toward tumorigenesis: Molecular targets of CDC25A in cell-cycle regulation. J Cell Biochem. 2019;120(3): 29192928.

25. Ray D, Terao Y, Fuhrken PG, Ma ZQ, DeMayo FJ, Christov K, et al. Deregulated CDC25A Expression Promotes Mammary Tumorigenesis with Genomic Instability. Cancer Res. 2007;67(3):984-91. 
26. Sengupta S, Jana S, Bhattacharyya A. TGF- $\beta$-Smad2 dependent activation of CDC 25A plays an important role in cell proliferation through NFAT activation in metastatic breast cancer cells. Cell Signal. 2014;26(2):240-252.

27.Brunetto E, Ferrara AM, Rampoldi F, Talarico A, Cin ED, Grassini G, et al. CDC25A protein stability represents a previously unrecognized target of HER2 signaling in human breast cancer: implication for a potential clinical relevance in trastuzumab treatment. Neoplasia. 2013;15(6):579-90.

28.Albert H, Santos S, Battaglia E, Brito M, Monteiro C, Bagrel D. Differential expression of CDC25 phosphatases splice variants in human breast cancer cells. Clin Chem Lab Med. 2011;49(10):1707-14.

29. Wang P, Zou FD, Zhang XD, Li H, Dulak A, Tomko RJ, et al. MicroRNA 21 negatively regulates CDC25A and cell cycle progression in colon cancer cells. Cancer Res. 2009;69(20):8157-65.

30. Butz H, Németh K, Czenke D, Likó I, Czirják S, Zivkovic V, et al. Systematic investigation of expression of $\mathrm{G} 2 / \mathrm{M}$ transition genes reveals $\mathrm{CDC} 25$ alteration in nonfunctioning pituitary adenomas. Pathol Oncol Res. 2017;23(3):633-641.

31. Rao PC, Begum S, Sahai M, Sriram DS. Coptisine-induced cell cycle arrest at G2/M phase and reactive oxygen species-dependent mitochondria-mediated apoptosis in non-small-cell lung cancer A549 cells. Tumour Biol. 2017;39(3):1010428317694565.

32. Olsvik HL, Lamark T, Takagi K, Larsen KB, Evjen G, Øvervatn A, et al. FYC01 Contains a C-terminally extended, LC3A/B-preferring LC3-interacting region (LIR) motif required for efficient maturation of autophagosomes during basal autophagy. J Biol Chem. 2015;290(49): 29361-74.

33. Dionne LK, Peterman E, Schiel J, Gibieža P, Skeberdis VA, Jimeno A, et al. FYCO1 regulates accumulation of post-mitotic midbodies by mediating LC3-dependent midbody degradation. J Cell Sci. 2017;130(23):4051-4062.

34. Sillars-Hardebol AH, Carvalho B, de Wit M, Postma C, Delis-van Diemen PM, Mongera S, et al. Identification of key genes for carcinogenic pathways associated with colorectal adenoma-to-carcinoma progression. Tumour Biol. 2010;31(2):89-96.

35. Eissa S, Matboli M, Awad N, Kotb Y. Identification and validation of a novel autophagy gene expression signature for human bladder cancer patients. Tumor Biology.

2017;39(4):1010428317698360.

36. Wang JH, Qu DF, An JH, Yuan GM, Liu YF. Integrated microarray analysis provided novel insights to the pathogenesis of glaucoma. Mol Med Rep. 2017;16(6): 8735-8746.

37. Li M, Chen Y, Yao JH, Lu SM, Guan Y, Xu YQ, et al. Genome-Wide Association Study of Smoking Behavior Traits in a Chinese Han Population. Front Psychiatry. 2020;11:564239. 
38. Zhao Y, Gao YK, Xu XD, Zhou JW, Wang H. Multi-omics analysis of genomics, epigenomics and transcriptomics for molecular subtypes and core genes for lung adenocarcinoma. BMC Cancer. 2021;21(1):257.

39. Slatore CG, Au DH, Littman AJ, Satia JA, White E. Association of nonsteroidal anti-inflammatory drugs with lung cancer: results from a large cohort study. Cancer Epidemiol Biomarkers Prev. 2009;18(4):12031207.

40. Dasari S, Tchounwou PB. Cisplatin in cancer therapy: molecular mechanisms of action. Eur J Pharmacol. 2014;740:364-78.

41. Ghosh S. Cisplatin: The first metal based anticancer drug. Bioorg Chem. 2019; 88:102925.

42. Kim JE, Lee SY, Jang M, Choi HK, Kim JH, Chen HY, et al. Coumestrol Epigenetically Suppresses Cancer Cell Proliferation: Coumestrol Is a Natural Haspin Kinase Inhibitor. Int J Mol Sci. 2017;18(10):2228.

43. Que ZJ, Zhou ZY, Luo B, Dong CS, Jiang Y, Li HG, et al. Jingfukang induces anti-cancer activity through oxidative stress-mediated DNA damage in circulating human lung cancer cells. BMC Complement Altern Med. 2019;19(1):204.

44. Platta CS, Greenblatt DY, Kunnimalaiyaan M, Chen H. Valproic Acid Induces Notch1 Signaling in Small Cell Lung Cancer Cells. J Surg Res. 2008;148(1):31-37.

45. Hubaux R, Vandermeers F, Cosse JP, Crisanti C, Kapoor V, Albelda SM, et al. Valproic acid improves second-line regimen of small cell lung carcinoma in preclinical models. ERJ Open Res. 2015;1(2):000282015.

\section{Figures}



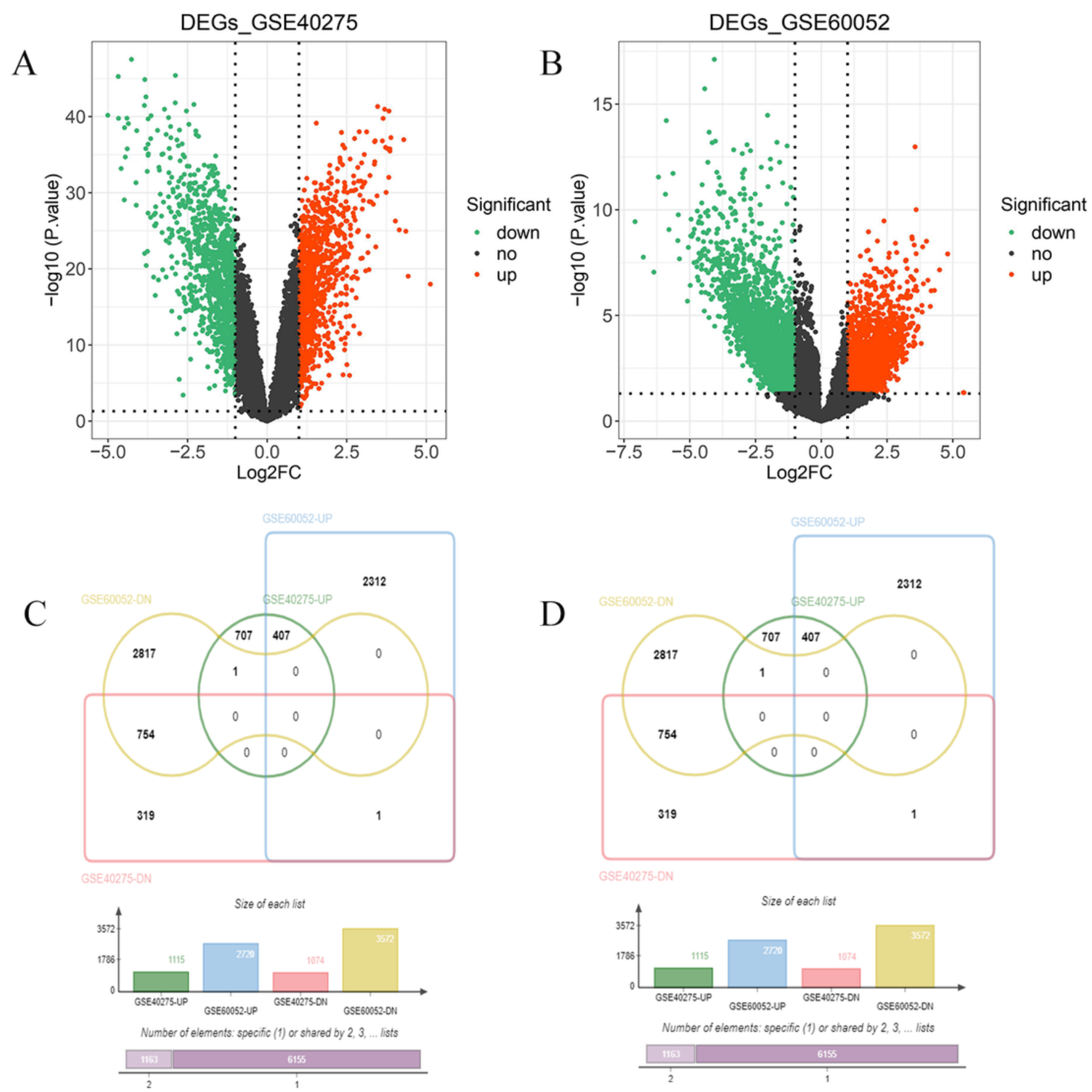

Figure 1

Identification of the DEGs in SCLC. (A) The volcano plot displayed the DEGs between SCLC and normal samples in the GSE40275 dataset. (B) The volcano plot displayed the DEGs between SCLC and normal samples in the GSE60052 dataset. (C) The co-DEGs of two datasets. (D) The intersection between coDEGs of two datasets and chr3 genes. 
A

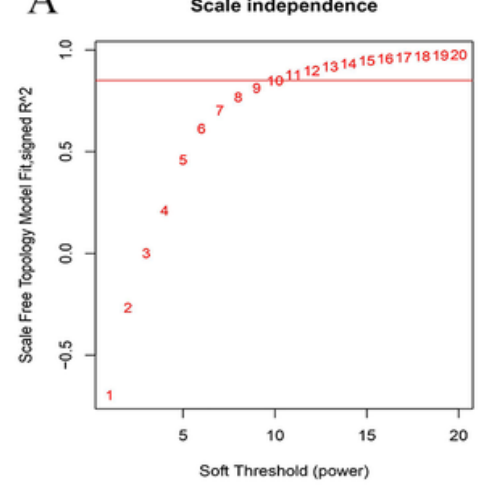

$\mathrm{C}$

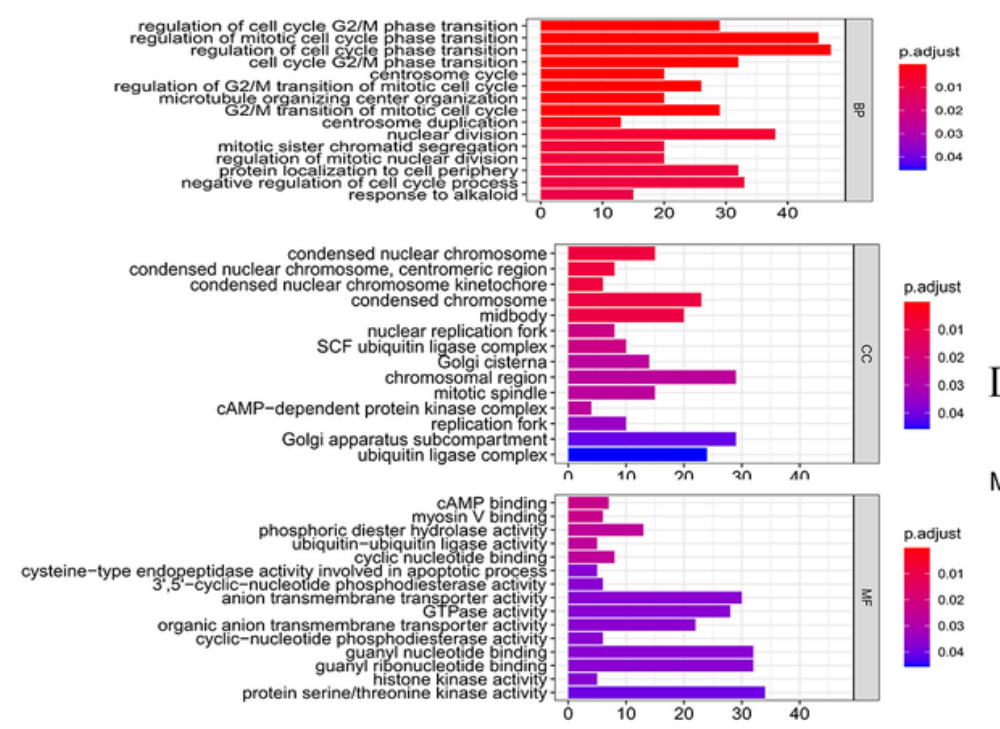

B

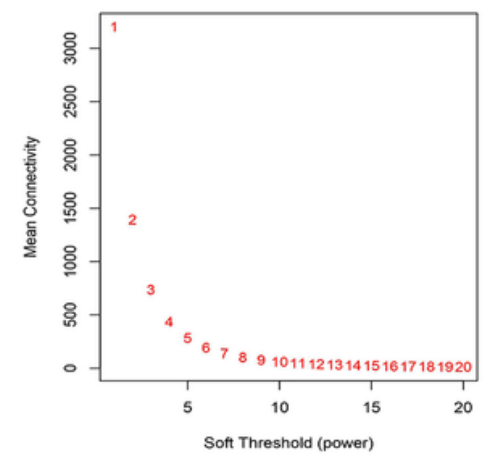

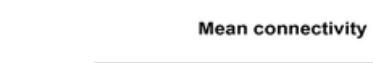

\begin{tabular}{|c|c|c|c|c|c|}
\hline MEdarkgrey & $\begin{array}{c}0.59 \\
(0.006)\end{array}$ & $\begin{array}{c}0.59 \\
(0.006)\end{array}$ & $\begin{array}{l}0.17 \\
(0.5)\end{array}$ & $\begin{array}{c}0.6 \\
(0.005)\end{array}$ & $\begin{array}{c}0.75 \\
(1 e-04)\end{array}$ \\
\hline MEgreenyellow & $\begin{array}{l}-0.2 \\
(0.4)\end{array}$ & $\begin{array}{l}0.25 \\
(0.3)\end{array}$ & $\begin{array}{c}0.5 \\
(0.03)\end{array}$ & $\begin{array}{c}-0.62 \\
(0.004)\end{array}$ & $\begin{array}{c}-0.12 \\
(0.6)\end{array}$ \\
\hline MEmagenta & $\begin{array}{c}0.46 \\
(0.04)\end{array}$ & $\begin{array}{c}-0.045 \\
(0.8)\end{array}$ & $\begin{array}{l}0.16 \\
(0.5)\end{array}$ & $\begin{array}{l}-0.31 \\
(0.2)\end{array}$ & $\begin{array}{l}0.33 \\
(0.2)\end{array}$ \\
\hline MEdarkgreen & $\begin{array}{l}-0.47 \\
(0.04)\end{array}$ & $\begin{array}{c}0.45 \\
(0.05)\end{array}$ & $\begin{array}{l}-0.2 \\
(0.4)\end{array}$ & $\begin{array}{l}0.19 \\
(0.4)\end{array}$ & $\begin{array}{l}0.34 \\
(0.1)\end{array}$ \\
\hline MEsaddlebrown & $\begin{array}{c}-0.9 \\
(6 e-08)\end{array}$ & $\begin{array}{l}-0.34 \\
(0.1)\end{array}$ & $\begin{array}{l}0.14 \\
(0.6)\end{array}$ & $\begin{array}{l}0.05 \\
(0.8)\end{array}$ & $\begin{array}{c}-0.58 \\
(0.007)\end{array}$ \\
\hline MEgrey60 & $\begin{array}{c}-0.21 \\
(0.4)\end{array}$ & $\begin{array}{c}0.48 \\
(0.03)\end{array}$ & $\begin{array}{c}-0.74 \\
(2 e-04)\end{array}$ & $\begin{array}{l}0.047 \\
(0.8)\end{array}$ & $\begin{array}{l}0.27 \\
(0.2)\end{array}$ \\
\hline MEsteelblue & $\begin{array}{c}-0.5 \\
(0.02)\end{array}$ & $\begin{array}{l}0.026 \\
(0.9)\end{array}$ & $\begin{array}{c}0.014 \\
(1)\end{array}$ & $\begin{array}{l}0.29 \\
(0.2)\end{array}$ & $\begin{array}{c}-0.21 \\
(0.4)\end{array}$ \\
\hline MEblack & $\begin{array}{c}-0.12 \\
(0.6)\end{array}$ & $\begin{array}{l}-0.52 \\
(0.02)\end{array}$ & $\begin{array}{l}0.093 \\
(0.7)\end{array}$ & $\begin{array}{c}-0.97 \\
(3 e-13)\end{array}$ & $\begin{array}{l}-0.52 \\
(0.02)\end{array}$ \\
\hline MEturquoise & $\begin{array}{c}-0.093 \\
(0.7)\end{array}$ & $\begin{array}{c}-0.24 \\
(0.3)\end{array}$ & $\begin{array}{c}-0.18 \\
(0.5)\end{array}$ & $\begin{array}{l}-0.42 \\
(0.06)\end{array}$ & $\begin{array}{c}-0.29 \\
(0.2)\end{array}$ \\
\hline MEbrown & $\begin{array}{c}-0.0036 \\
\text { (1) }\end{array}$ & $\begin{array}{c}-0.62 \\
(0.003)\end{array}$ & $\begin{array}{c}0.2 \\
(0.4)\end{array}$ & $\begin{array}{c}0.3 \\
(0.2)\end{array}$ & $\begin{array}{l}-0.44 \\
(0.05)\end{array}$ \\
\hline MEdarkorange & $\begin{array}{l}0.33 \\
(0.2)\end{array}$ & $\begin{array}{l}-0.39 \\
(0.09)\end{array}$ & $\begin{array}{l}-0.46 \\
(0.04)\end{array}$ & $\begin{array}{l}0.17 \\
(0.5)\end{array}$ & $\begin{array}{l}0.28 \\
(0.2)\end{array}$ \\
\hline \multirow[t]{2}{*}{ MEgrey } & $\begin{array}{c}-0.18 \\
(0.4)\end{array}$ & $\begin{array}{c}-0.0028 \\
\text { (1) }\end{array}$ & $\begin{array}{l}0.23 \\
(0.3)\end{array}$ & $\begin{array}{c}-0.0037 \\
\text { (1) }\end{array}$ & $\begin{array}{c}-0.16 \\
(0.5)\end{array}$ \\
\hline & 仓̂ & pे & $p^{8}$ & $s^{e^{+}}$ & \\
\hline
\end{tabular}

D

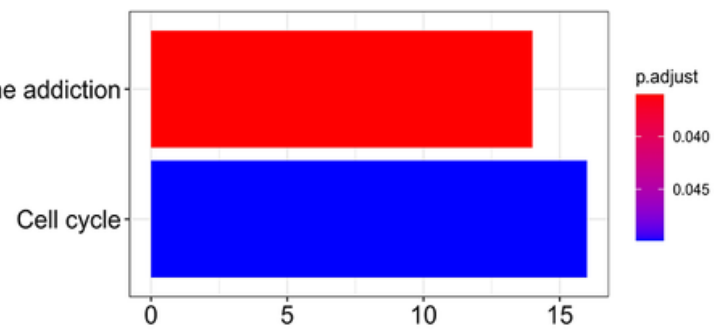

\section{Figure 2}

Identification of hub genes and their functional annotation. (A) The Soft Threshold of Scale independence and Mean connectivity by WGCNA. (B) The relationships between twelve co-expressed modules and clinical characteristics. (C) GO-BP, GO-CC, and GO-MF analysis revealed that these hub genes were significantly enriched in various cell cycle-related terms. (D) KEGG pathway prompted that the cell cycle pathway was significantly enriched. 

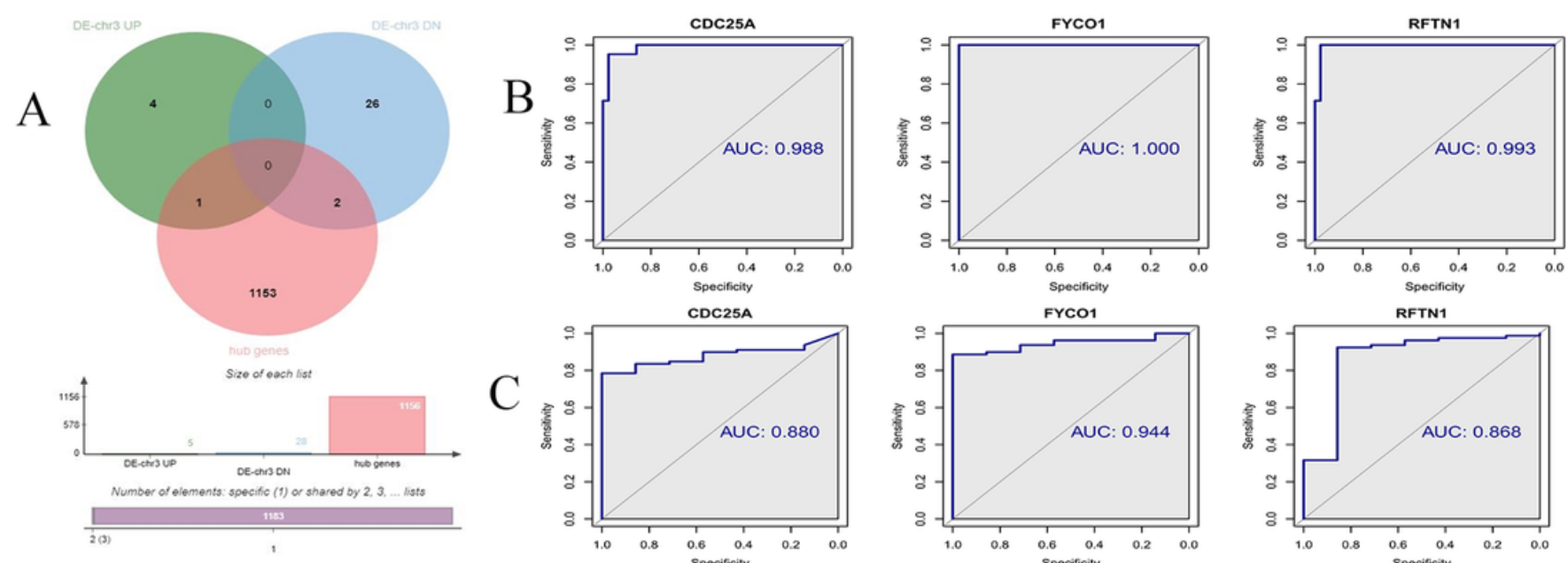

C

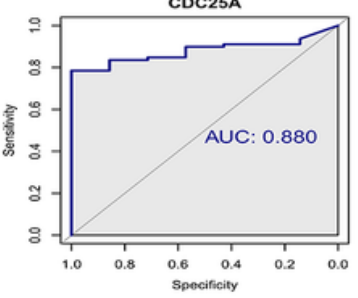

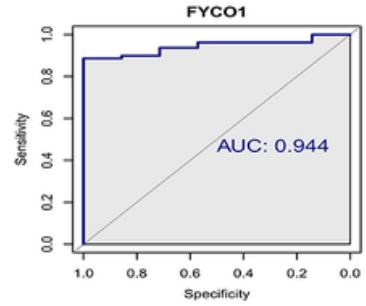

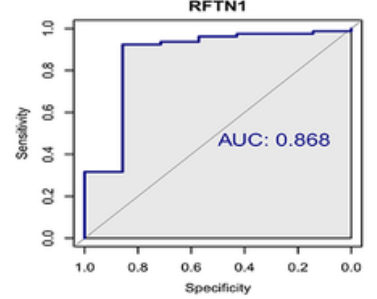

GSE40275

Type 审 Normal 安 SCLC

GSE60052

Type 审 Normal 审 SCLC
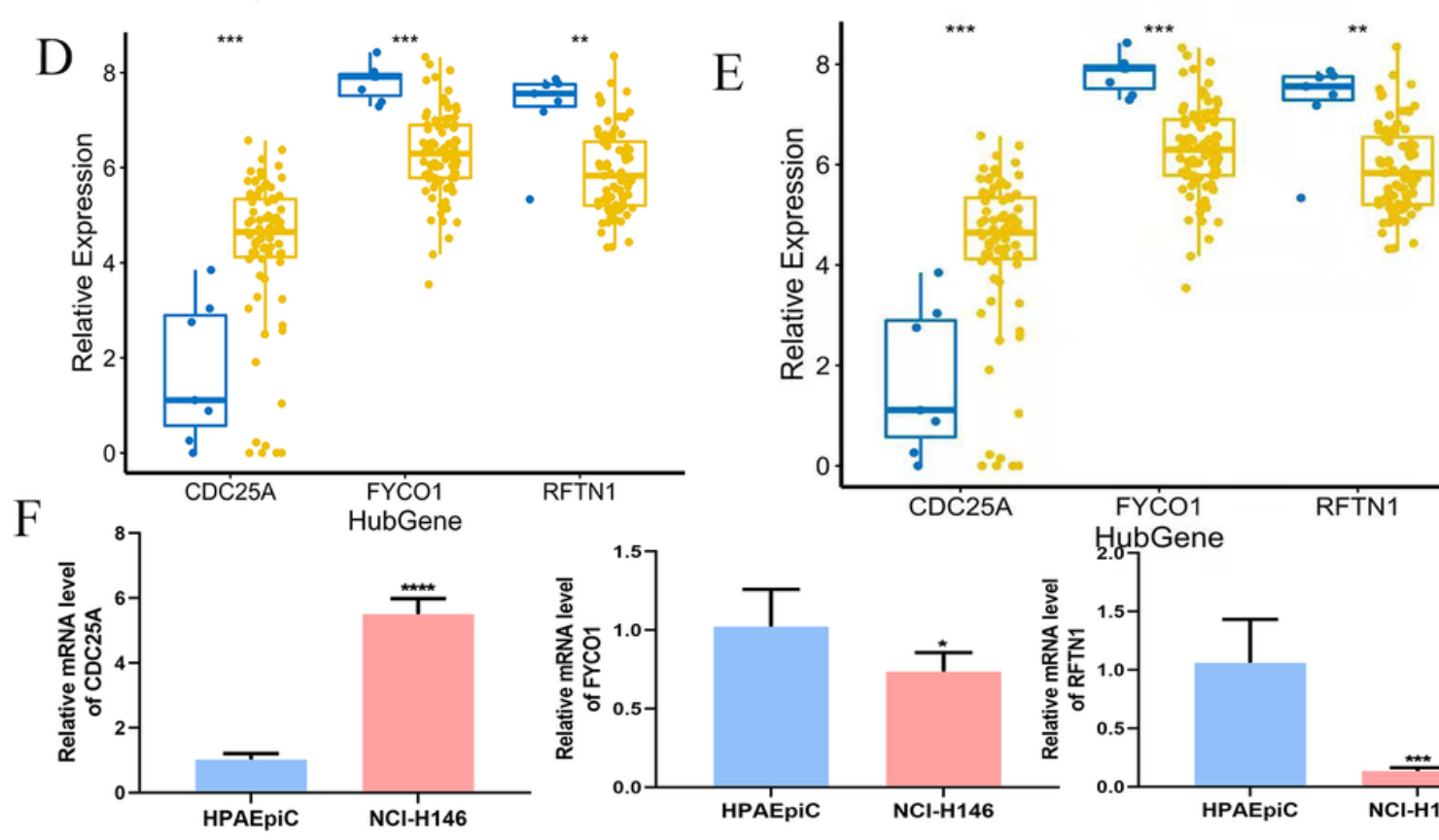

\section{Figure 3}

Identification of the 3 overlapping genes for the DE-chr3 genes and the hub genes in SCLC. (A) The Venn figure displayed the 3 overlapping genes for the DE-chr3 genes and the hub genes. (B) The ability of the 3 overlapping genes to discriminate between normal and SCLC samples in the GSE40275 dataset was assessed by ROC curves. (C) The ability of the 3 overlapping genes to discriminate between normal and SCLC samples in the GSE60052 dataset was assessed by ROC curves. (D) The expression patterns of these 3 overlapping genes in the GSE40275 dataset. (E) The expression patterns of these 3 overlapping genes in the GSE60052 dataset. (F) qRT-PCR results showed the relative mRNA level of these 3 overlapping genes between $\mathrm{NCl}-\mathrm{H} 146$ and HPAEpiC. 


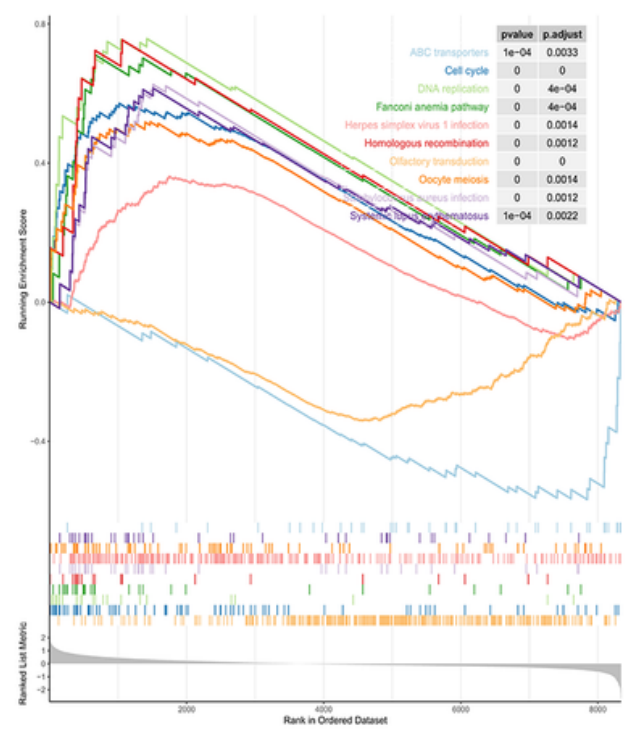

A

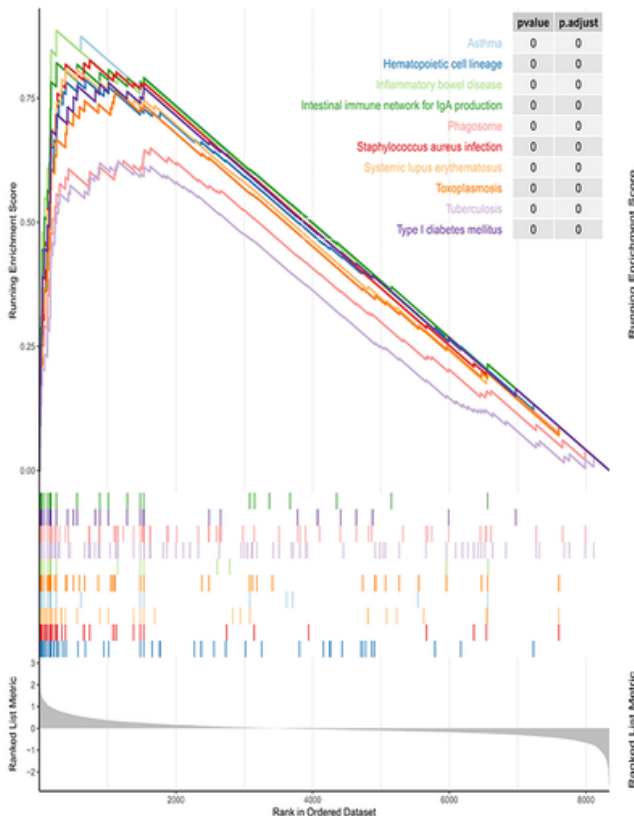

B

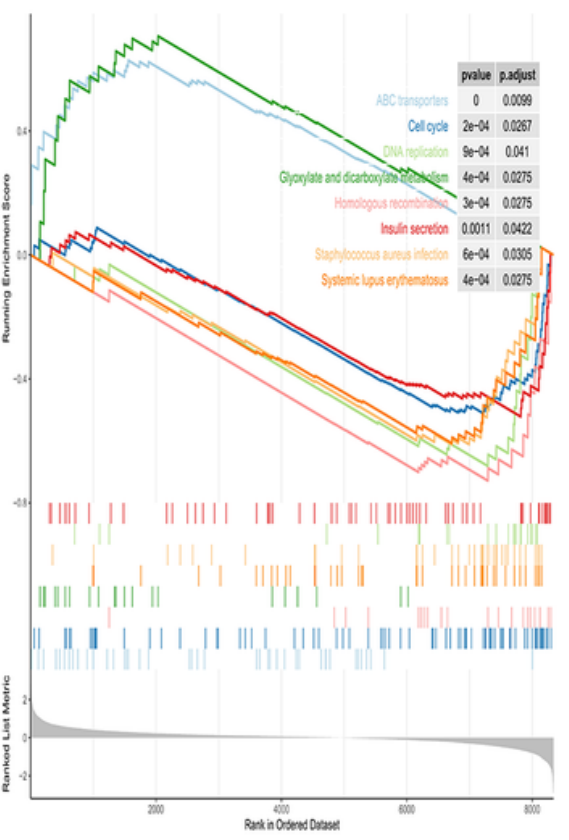

$\mathrm{C}$

Figure 4

Functional annotation of each diagnostic biomarker. (A) The Single-gene GSEA results revealed that CDC25A was enriched to a total of 22 KEGG pathways. (B) The Single-gene GSEA results revealed that RFTN1 was significantly associated with 52 KEGG pathways. (C) The Single-gene GSEA results revealed that FYCO1 was mainly involved in 8 KEGG pathways. A merged enrichment plot from Single-gene GSEA including enrichment score and gene sets.

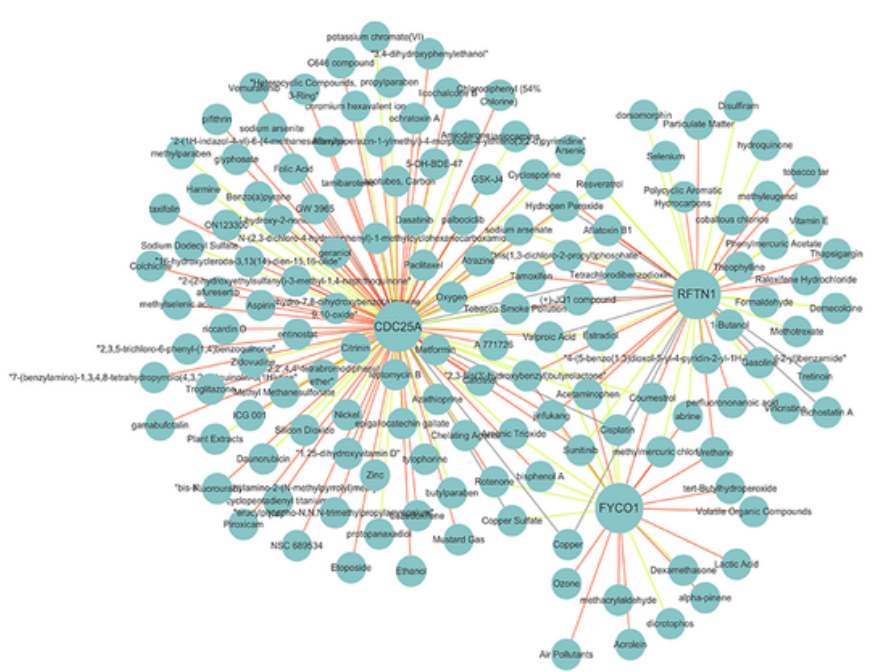

A

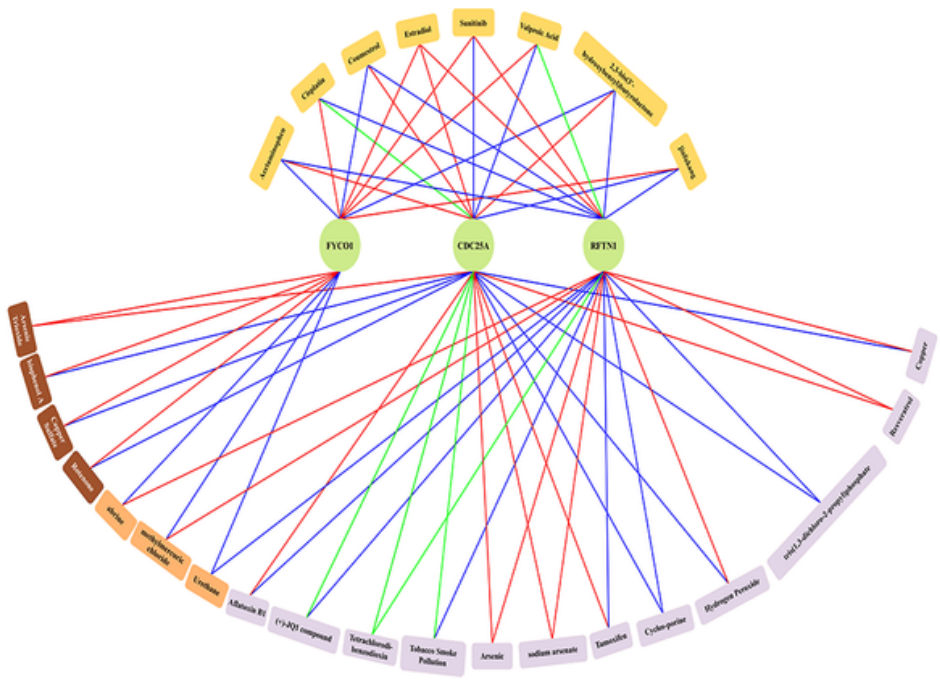

B

Figure 5 
Pharmaceutical prediction for regulating the expression of diagnostic biomarkers by CTD. (A) A complete drug-diagnostic biomarker network was constructed using Cytoscape. (B) The regulatory relationships of 27 shared drugs (drugs shared by 2 diagnostic biomarkers and drugs shared by all 3 diagnostic biomarkers) with diagnostic biomarkers.

\section{Supplementary Files}

This is a list of supplementary files associated with this preprint. Click to download.

- SupplementaryTable1.xlsx

- SupplementaryTable2.xlsx

- SupplementaryTable3.xIsx

- SupplementaryTable4.xIsx

- SupplementaryTable5.xlsx

- SupplementaryTable6.xlsx

- SupplementaryTable7.xlsx

- SupplementaryTable9.xIsx

- SupplementaryFigure1.png

- SupplementaryFigure3.png

- SupplementaryTable11.xlsx

- SupplementaryTable12.xIsx

- SupplementaryTable13.xlsx

- SupplementaryTable14.xIsx

- SupplementaryTable15.xIsx

- SupplementaryTable16.xlsx

- SupplementaryTable10.xIsx

- SupplementaryFigure2.png

- SupplementaryTable8.xlsx

- SupplementaryTable7.xlsx 